\title{
Falsifying mental models: Testing the predictions of theories of syllogistic reasoning
}

\author{
STEPHEN E. NEWSTEAD, SIMON J. HANDLEY, and ED BUCK \\ University of Plymouth, Plymouth, England
}

\begin{abstract}
Four experiments are reported that tested the claim, drawn from mental models theory, that reasoners attempt to construct alternative representations of problems that might falsify preliminary conclusions they have drawn. In Experiment 1, participants were asked to indicate which alternative conclusion(s) they had considered in a syllogistic reasoning task. In Experiments 2-4, participants were asked to draw diagrams consistent with the premises, on the assumption that these diagrams would provide insights into the mental representation being used. In none of the experiments was there any evidence that people constructed more models for multiple-model than for single-model syllogisms, nor was there any correlation between number of models constructed and overall accuracy. The results are interpreted as showing that falsification of the kind proposed by mental models theory may not routinely occur in reasoning.
\end{abstract}

To what extent do people reason when they are given reasoning tasks to carry out? This apparently trivial question is actually an important and currently controversial one. Some theorists claim that people solve reasoning problems by utilizing sophisticated general-purpose reasoning rules; others suggest that people carry out semantic processes that contain a reasoning element in them; while still others believe that people use no reasoning at all, but instead base their responding on surface characteristics of the problem presented. What is more, all of these theories have vigorous current proponents; in each case, important theoretical developments have taken place within the past 2 or 3 years.

Rips (1994) is the most vociferous current champion of the claim that there exist general-purpose inference rules. The essence of this claim is that people solve reasoning problems by putting the premises into an abstract propositional form, applying appropriate inference rules, and then producing the conclusion generated. In this theory, reasoning errors are explained by the absence of an appropriate rule, by the sheer length of the inference process involved, or by failure to encode the premises correctly.

Johnson-Laird and Byrne (1991) have proposed that reasoning uses the same processes as comprehension, in that the first stage is to construct a mental model of the premises. Mental models are abstract representations of information that preserve many of the properties and relationships of the entities described. Reasoning occurs primarily when there is a subsequent attempt to falsify conclusions generated from the model constructed. This is

We thank Thad Polk, Steve Smith, and two anonymous reviewers for their helpful comments on an earlier version of this paper. Correspondence should be addressed to S. E. Newstead, Department of Psychology, University of Plymouth, Drake Circus, Plymouth PL4 8AA, England (e-mail: snewstead@plym.ac.uk). done by attempting to construct alternative models in which the conclusion no longer holds true.

The claim that no reasoning at all takes place is an old one. Janis and Frick (1943) presented evidence that people will often respond on the basis of their prior beliefs and prejudices rather than on the logic of the problem presented. Even earlier than that, Woodworth and Sells (1935) suggested that, in syllogistic reasoning, people respond on the basis of the "mood" of the quantifiers presented in the premises, not on the basis of logical analysis of the problem. The modern heir to this tradition is rational choice theory, which claims that people respond on the basis of the informativeness of the premises, which is independent of any logical analysis of the problem (see, e.g., Oaksford \& Chater, 1994).

The focus of the present paper is the mental models approach and in particular its adequacy as an explanation of syllogistic reasoning behavior. Syllogisms are problems with two premises and a conclusion, each of which contains one of the quantifiers "all," "no," "some," and "some ... not." One of the terms, the middle term, is repeated in the two premises, and the task is to draw a conclusion linking the other two terms. The terms in the premises can be presented in different orders, which correspond to the different figures of the syllogism.

The theory of mental models was first developed in the area of syllogistic reasoning and hence this is an appropriate testing ground for its adequacy. The theory claims that internal models are built containing tokens corresponding to elements mentioned in the problem. For example, the syllogism "Some A are B; all B are C" could be represented as follows:

$$
\text { a }\left[\begin{array}{ll}
{[b]} & c]
\end{array}\right.
$$

The small letters are tokens representing individuals described by the syllogism, and the square brackets indicate that the enclosed elements are exhaustively repre- 
sented in the model (see Johnson-Laird \& Byrne, 1991, for a fuller account of the notation used). The number of entities of each kind that are entered in the model is arbitrary; in the model presented above, there is just one of each type, but more could have been represented. This model thus indicates that all possible $b$ s have been represented and that these are also $c \mathrm{~s}$, but that there might be $a \mathrm{~s}$ and $c$ s that are not $b \mathrm{~s}$. Having constructed such a model, the reasoner can draw the provisional conclusion that "Some $\mathrm{A}$ are C." The reasoner then tries to falsify this conclusion by constructing alternative models in which the conclusion does not hold. In this case, the search for such alternative models fails, since however the premises are construed, at least one of the $a$ s is a $c$; hence the conclusion can be validly drawn.

In other cases, however, more than one model can be constructed. The syllogism "No A are B; all B are C" permits all the following models:
(1)

[a]

[a]

[[b] c]

$[$ [b] c]
(2)

[a]

[a]

$\left[\begin{array}{ll}{[\mathrm{b}]} & \mathrm{c}]\end{array}\right.$

[[b] c]
(3)

[a]

[a]

[b] c]

In each of these models, there is no overlap between $a \mathrm{~s}$ and $b s$, but the way in which the $c s$ are represented is crucial. In Model 1, none of the $c$ s are $a$ s; in Model 2, some of them are; and in Model 3, all of them are. In order to draw the logically correct conclusion, "Some $\mathrm{C}$ are not A," reasoners need to construct all three models. Having constructed the first model, the conclusion "No A are C" can be drawn, but this is falsified by the second model; and the conclusion "Some A are not C," compatible with the first two models, can be eliminated only by constructing the third model.

One of the central questions in this area is whether falsification takes place. In other words, having constructed a model of the situation described and generated a conclusion consistent with it, do people then try to construct alternative models in an attempt to disconfirm the putative conclusion? This assumption is at the heart of mental models theory. Indeed, the difficulty inherent in constructing alternative models is the basic mechanism for explaining why multiple-model syllogisms are so much more difficult than single-model ones. On the other hand, Polk and Newell's (1995) verbal reasoning theory, which is in many ways similar to mental models theory, differs in that it does not assume that falsification takes place. This is more than a trivial disagreement over emphasis. Falsification, if it occurs, is the reasoning process. If it is removed from the mental models approach, then this becomes principally a way of explaining representation only. The essence of Polk and Newell's theory is that representational processes alone can explain reasoning performance-this is why they call it a verbal reasoning theory.

The evidence as to whether falsification takes place is equivocal. One of the main predictions it generates is that multiple-model syllogisms should be more difficult than single-model ones. The evidence that this is so is overwhelming, not only in syllogistic reasoning but also in a host of other reasoning tasks (Johnson-Laird \& Byrne, 1991). However, this is not conclusive evidence that falsification occurs, since a number of other explanations can be put forward for this finding. Evans, Newstead, and Byrne (1993) pointed out that a number of other theories can also explain the observed difficulty of different syllogisms even though they do not evoke the concept of mental models to do so.

Second, mental models theory itself can explain the relative difficulty of syllogisms without using the notion of falsification. If it is assumed that people construct just one mental model from the premises and then draw a conclusion consistent with it, then multiple-model syllogisms will be more difficult than single-model ones. This is because with multiple-model syllogisms, a participant might construct a model that leads to a conclusion other than that dictated by logic. With single-model syllogisms, this cannot occur. Hence the findings can be explained quite simply in terms of the probability of constructing a model from which the correct conclusion follows. What this approach does not explain is how people ever decide that no valid conclusion follows.

Very little research has investigated directly whether falsification occurs. Polk and Newell (1995) carried out extensive statistical modeling of existing data obtained from syllogistic reasoning tasks. One of the parameters they used in their modeling was whether or not falsification took place. They found that it explained very little of the individual variance, since fewer than $2 \%$ of the best fitting settings included this parameter.

A different conclusion is suggested, however, in the research of Byrne and Johnson-Laird (1990). They gave participants a syllogistic reasoning task and then tested their recognition memory for the conclusions they had produced. The authors argued that the recognition errors made were likely to involve conclusions that were suggested by other models of the premises. It is assumed that, when solving the syllogisms, participants generate a number of possible models and conclusions and that some of these are likely to intrude in the recognition memory phase of the experiment. Byrne and Johnson-Laird's data bore out this prediction: When participants who had correctly solved the syllogism made a recognition error in remembering the conclusion they had drawn, this error always involved giving a conclusion consistent with one of the models they had supposedly constructed.

Unfortunately, this result is not as compelling as it first seems. During the recognition phase of the study, Byrne and Johnson-Laird (1990) re-presented the original syllogism. It is thus possible that participants were not using their recognition memory to carry out the task but were instead re-solving the syllogism. The conclusion they "remembered" will inevitably be consistent with one of the possible mental models resulting from the premises, but it does not follow that the conclusion they gave was generated during the initial phase of the experiment. Byrne and 
Johnson-Laird carried out a follow-up study suggesting that participants were indeed solving the problems afresh: When the recognition task was given without re-presenting the premises, memory for conclusions was random.

Other evidence pertinent to the existence of falsification in syllogistic reasoning can be found in research on the effects of belief bias. It has been demonstrated many times that people are more likely to accept a conclusion if it is believable than if it is unbelievable, and that this effect is more marked with invalid than with valid syllogisms (see, for a review, Evans et al., 1993). Mental models theory can provide a persuasive account of many of the findings (e.g., Newstead, Pollard, Evans, \& Allen, 1992; Oakhill, Johnson-Laird, \& Garnham, 1989). Central to this account is the assumption that believability can drive the search for counterexamples (i.e., falsification). Note, however, that this account does not assume that falsification always occurs; it would be compatible with this account to claim that falsification does not occur as a matter of routine and indeed is likely to occur only if the initial model produces an unbelievable conclusion.

Although Byrne and Johnson-Laird's (1990) methodology may have been flawed, their rationale seems a sound one. If people do construct several mental models and generate conclusions consistent with each of them, then there should be evidence of this in their cognitive processes. Byrne and Johnson-Laird chose to look for evidence of such conclusions in memory for the conclusions, but it is also feasible to look for more immediate evidence - that is, during or immediately after the inferential process. Experiment 1 took a very direct approach to this question by asking participants immediately after they had solved a syllogism which conclusion(s) they had considered. The prediction was straightforward: More conclusions would be considered following multiplemodel than following single-model syllogisms.

\section{EXPERIMENT 1}

\section{Method}

Participants. Twenty participants took part in this study. All were Ist-year undergraduate psychology students from the University of Plymouth who received course credit for their participation.

Materials. The study used 24 syllogisms, 6 drawn from each of the four syllogistic figures. Within each figure, there were two single-model, two multiple-model, and two indeterminate syllogisms, as described by Johnson-Laird and Bara (1984). The content of the syllogisms was names of occupations. To illustrate, one of the singlemodel syllogisms in figure ab-bc was "All of the buskers are computer operators. None of the computer operators are boxers." An indeterminate syllogism in figure ab-cb was "All of the inspectors are dancers. Some of the welders are dancers." Different content was used in each syllogism, and every effort was made to ensure that no related occupations were used within the same syllogism. In order to protect against the possibility that, despite these precautions, there were still expectancy or belief effects associated with the terms used, a second set of syllogisms was constructed from the same terms but in different combinations.

Procedure. The syllogisms were presented in a booklet. The instructions at the front of the booklet indicated that the task was to draw a conclusion linking the two end terms of the syllogism using one of the conclusions "all," "no," "some," and "some . . not," or to indicate that no valid conclusion followed. An example of a syllogism and of the possible conclusions that could be generated from it was given. The instructions also explained what syllogisms were: that the premises should be assumed to be true; that the conclusion must follow with certainty if it was to be written down; that "some" does not preclude "all"; and that the strongest conclusion possible should be drawn. It was also suggested that it might be useful to imagine the premises as referring to a group of people in a room.

Participants were required to solve the 24 syllogisms in turn. Before drawing their conclusion, they were reminded that their answer should contain one of the quantifiers-"some," "all," "no," or "some ... not"- and that it should link the two end terms in either direction. The syllogisms were presented in different random orders for each participant. Half the participants were presented with the first set of occupations, the other half with the second set.

Immediately after drawing a conclusion to a syllogism, participants were required to indicate, on a separate piece of paper, how many possible answers they had considered before making their final decision. They were presented with a list of the nine possible conclusions, including "no valid conclusion." They were asked to place a tick beside any of the conclusions that they had considered before coming to their final decision. They were asked to tick a conclusion if they had thought about it as a possible conclusion while solving the syllogism. After carrying out this part of the task, they proceeded to the next syllogism.

\section{Results}

Two sets of data were collected: response accuracy and the number of conclusions considered. The accuracy data are presented in Table 1. An analysis of variance (ANOVA) revealed no main effect of figure $[F(3,57)=2.40, p>.05]$, a main effect of number of models $[F(2,38)=46.2, p<$ $.001]$, and no interaction between these two variables $[F(6,114)=.34, p>.05]$. Breakdown of the main effect of number of models revealed that single-model syllogisms were associated with significantly greater accuracy than either multiple-model or indeterminate syllogisms, but that there was no difference between the latter two. (In these and all other breakdown analyses reported in this paper, we used planned $F$-test comparisons with an alpha level of .01).

The data on number of considered conclusions as a function of number of models can be found in Table 2 . Considered conclusions were those that the participant ticked, except when this was the conclusion actually given; such responses were ignored in the analysis. An ANOVA indicated no significant main effect of number of models $[F(2,38)=.22, p>.05]$. Exactly the same picture emerges if only syllogisms to which participants gave the correct response are included, as can be seen from the figures in the bottom row of Table 2 . The latter data were not submitted to more formal analysis because of the number of missing data points, especially in the multiplemodel condition, in which so few correct responses were given.

It might be expected that those participants who produced many provisional conclusions would tend to be more accurate on the syllogism task. This was tested by correlating the number of conclusions considered against overall accuracy. The Pearson correlation was actually 
Table 1

Proportion Correct as a Function of Figure and Number of Models in Experiment 1

\begin{tabular}{|c|c|c|c|c|c|c|c|}
\hline Figure & Syllogism & Single Model & Syllogism & Multiple Model & Syllogism & Indeterminate & Figure $M$ \\
\hline \multirow[t]{2}{*}{$a b-b c$} & IabAbc & .70 & IabEbc & .15 & Aablbc & .10 & \\
\hline & AabEbc & .70 & EabIbc & .10 & EabObc & .25 & .34 \\
\hline \multirow[t]{3}{*}{$\mathrm{ba}-\mathrm{cb}$} & Abalcb & .75 & IbaEcb & .10 & IbaAcb & .05 & \\
\hline & EbaAcb & .50 & EbaIcb & .05 & EbaEcb & .30 & \\
\hline & & & & & & & .29 \\
\hline \multirow{2}{*}{$a b-c b$} & $\begin{array}{l}\text { AabAco } \\
\text { AabEcb }\end{array}$ & $\begin{array}{l}.53 \\
.75\end{array}$ & EabIcb & $\begin{array}{l}.10 \\
.10\end{array}$ & AabIcb & .15 & \\
\hline & & & & & & & .30 \\
\hline \multirow[t]{3}{*}{$b a-b c$} & IbaAbc & .80 & Ebalbc & .15 & IbaObc & .20 & \\
\hline & Abalbc & .85 & IbaEbc & .15 & $\mathrm{EbaObc}$ & .30 & \\
\hline & & & & & & & .42 \\
\hline$M$ & & .70 & & .12 & & .19 & \\
\hline
\end{tabular}

(nonsignificantly) negative $(r=-.17)$. Separate correlations were calculated for single-model, multiple-model, and indeterminate syllogisms, but these were also all negative (though none were statistically significant).

Also of interest are the actual conclusions considered. Byrne and Johnson-Laird (1990) placed great store on the provisional conclusions reached on indeterminate syllogisms on which the participant correctly responded that no valid conclusion followed. The average number of conclusions considered for such syllogisms was 1.10 almost exactly the same as the overall mean for all syllogisms (Table 2). Although all of the conclusions indicated were compatible with putative models that could be constructed from the premises, this is not strong evidence in support of mental models theory since the great majority of possible conclusions were compatible with the premises.

Participants also responded that no valid conclusion followed to syllogisms for which this was not the correct response. Since "no valid conclusion" responses are assumed to occur when reasoners have searched a number of different models for a possible conclusion, one might have expected that participants would consider many conclusions before deciding that none followed. In fact, when erroneously giving "no valid conclusion" responses, participants indicated on average that they had considered only .66 conclusions - rather fewer than were indicated prior to other conclusions being given!

\section{Discussion}

The results indicate quite clearly that there was no difference in the number of conclusions considered be- tween single-model, multiple-model, and indeterminate syllogisms. If participants had been attempting to construct models to falsify their initial conclusions, they clearly should have considered more conclusions with the multiple-model and indeterminate syllogisms than with the single-model ones. This would be expected to be particularly marked on correctly answered syllogisms since the construction of alternative models is seen as crucial to a correct solution. The finding that this was not the case inevitably calls into question the existence of attempted falsification of the kind proposed by mental models theory.

This does not mean that participants did not consider alternative conclusions to the one they selected. On the contrary, our evidence suggests that people frequently consider and then reject one or more alternative conclusions. However, they are no more likely to do this with multiple-model syllogisms than with single-model ones. Even when only a single model has been constructed, more than one conclusion is possible. For example, whenever a model is constructed in which "Some A are B" is true, then "Some B are A" will also be necessarily true, and other conclusions may be possible as well.

Other evidence from the present experiment also calls into question some of the assumptions of mental models theory. There was no correlation between the number of conclusions considered and overall accuracy, as might be expected if these conclusions indicated that participants are actively considering a conclusion derived from a preliminary mental model. Nor was there any evidence that reasoners responded that no valid conclusion followed only after considering a variety of possible models. If any-

Table 2

Number of Conclusions Considered

as a Function of Number of Models in Experiment 1

\begin{tabular}{lcccr}
\hline & Single Model & Multiple Model & Indeterminate & $M$ \\
\hline All syllogisms & 1.05 & 1.12 & 1.12 & 1.09 \\
Correct syllogisms only & 1.04 & 1.11 & 1.10 & 1.06 \\
\hline
\end{tabular}


Table 3

Proportion Correct as a Function of Experimental Condition in Experiment 2

\begin{tabular}{lccc}
\hline & \multicolumn{3}{c}{ Model } \\
\cline { 2 - 4 } Condition & Single & Multiple & Indeterminate \\
\hline Experimental & .66 & .18 & .34 \\
Control & .87 & .28 & .28 \\
\hline
\end{tabular}

thing, such responses seemed to be preceded by the consideration of fewer possible conclusions than did other responses. The present experiment does not indicate how "no valid conclusion" responses are reached, but the evidence suggests that it is not by ruling out other alternative models that have been considered.

However, there are potential criticisms of the present experiment. Although the present way of determining considered conclusions was more direct than Byrne and Johnson-Laird's (1990) memory task, it was nevertheless carried out after the reasoning task had been completed. This brings with it a number of problems, including that of memory load. Solving syllogisms is a demanding task, and participants may utilize all available memory in this task; hence they might forget some of the intermediate stages involved in their reasoning and be unable to recall with any accuracy conclusions they have considered and rejected. The fact that participants indicated on average that they had considered more than one alternative conclusion for each syllogism argues against this, but it remains a possibility.

Hence it was decided to use an alternative, and arguably more direct, measure of the representations that reasoners construct from the premises. This was done in Experiment 2 by asking participants to draw diagrams consistent with the syllogistic premises with which they had been presented. It was assumed that such diagrams would provide insight into the kinds of representations that participants were using. Three predictions can be made on the basis of mental models theory: first, that more conceptually distinct diagrams will be constructed for multiple-model and indeterminate syllogisms than for single-model ones; second, that, for multiple-model and indeterminate syllogisms, the more different diagrams that are constructed, the higher the accuracy will be; and third, that requiring people to construct diagrams should lead to improved performance over a control group required only to solve the syllogisms.

\section{EXPERIMENT 2}

\footnotetext{
Method

Participants. There were 62 participants, all of whom were 1styear undergraduate psychology students from the University of Plymouth who received course credit for their participation.

Materials. Six syllogisms were used in this study, all of which were of the form ab-bc. There were two single-model, two multiplemodel, and two indeterminate syllogisms. The material used in the syllogisms consisted of shapes (squares or circles), patterns (striped or cross-hatched), and borders (bold or faint). Shape always corresponded to term $A$ of the syllogism, border always corresponded to
}

term $B$ (the middle term), and pattern always corresponded to term $C$. For example, the IabAbc syllogism was: "Some of the squares have bold borders; all of the bold bordered shapes are striped."

Procedure. There were two conditions in the experiment. A control group of 31 participants was presented with a paper booklet containing the six syllogisms in random order. Their task was simply to solve the syllogisms, having been given instructions similar to those used in Experiment 1.

A second, experimental group was required to carry out the more complex task of drawing three sets of six shapes consistent with each syllogism. Participants in this group were presented with the syllogism at the top of the page. Below this were three empty grids of six squares. In each square of each grid, they were asked to draw a shape using one of the 12 shape/border/pattern combinations described above. The participant's task was to draw three sets of shapes in such a way that each set of six shapes was consistent with the two premises of the syllogism. Participants were asked to make each set different. There was a reminder of the 12 shapes to choose from at the bottom of every page. Two pens of different thickness were provided to facilitate the drawing of bold and faint borders.

On completion of the three sets of shapes, participants were required to solve the syllogism in the same way as the control group.

\section{Results}

Two sets of data were collected: accuracy data for the control condition and for the experimental condition, and data concerning the sets of pictures drawn in the experimental condition. The accuracy data for the two conditions are shown in Table 3 . An ANOVA revealed a significant main effect of number of models $[F(2,120)=$ $56.4, p<.001]$; accuracy was significantly greater on single-model syllogisms than on either multiple-model or indeterminate syllogisms, but there was no significant difference between the latter. There was no significant difference in accuracy between participants who constructed models and those who did not $[F(1,60)=1.77, p>.1]$. The interaction between this variable and number of models was just significant $[F(2,120)=3.15, p<.05]$. Participants performed slightly better in the experimental condition on indeterminate problems, and slightly worse on valid syllogisms, whether single or multiple model.

The pictures drawn by participants can be depicted using mental model-like representations produced by mapping the properties contained in the diagrams onto token-based representations. For example, given the syllogism "Some of the squares have bold borders; all of the bold bordered shapes are striped," a participant might draw (1) a square with a striped bold border; (2) a square with a faint, unstriped border; (3) a circle with a striped bold border, (4) a circle with a faint striped border; (5) a circle with a faint, unstriped border; and (6) a circle with a faint, cross-hatched border. The following representation captures these relationships, where $a=$ square, $b=$ bold border, and $\mathrm{c}=$ striped.

$$
\begin{array}{lll}
a & & \\
\mathrm{a} & \mathrm{b} & \mathrm{c} \\
& \mathrm{b} & \mathrm{c} \\
& & \mathrm{c}
\end{array}
$$

The first line captures the fact that there are squares that do not have bold borders or stripes (diagram 2); the 

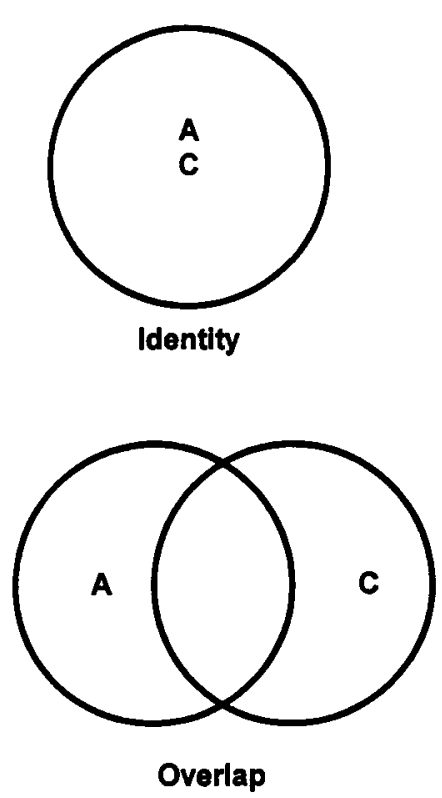

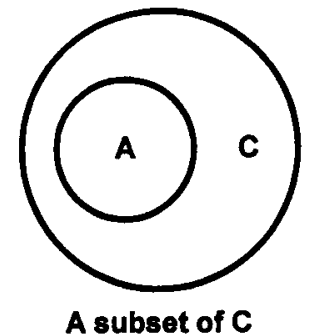

A subset of C
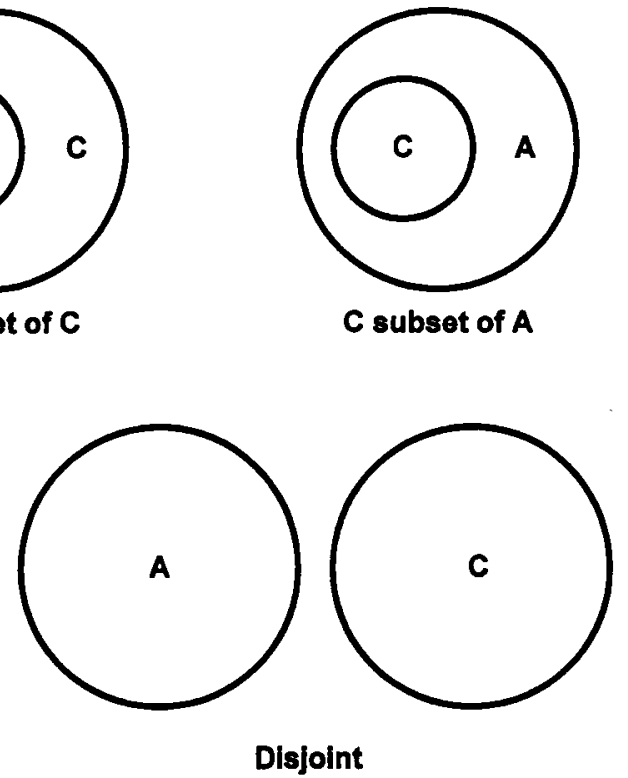

Figure 1. Euler circle representations of the relationship between two sets, $A$ and $C$.

second line that there are squares that have bold borders and stripes (diagram 1); the third that there are nonsquares that have bold borders and stripes (diagram 3); and the fourth that there are striped shapes that are not square and do not have bold borders (diagram 4). Diagrams containing none of the properties in the syllogism (5 and 6) are not represented.

Although these representations are similar to mental models, they are not identical. Mental models of the type described by Johnson-Laird and Byrne (1991) cannot be produced since there is no way of knowing whether entities have been exhaustively represented in the diagrams chosen.

Inspection of this representation readily reveals that there are $a \mathrm{~s}$ that are $c \mathrm{~s}$, but that there are other $a \mathrm{~s}$ that are not $c \mathrm{~s}$, and other $c \mathrm{~s}$ that are not $a \mathrm{~s}$. This means that it is valid to conclude that "Some A are C," "Some C are A," "Some A are not C," and "Some C are not A." This is equivalent to claiming that there is an overlap relationship between $A$ and $C$. In fact, all of the sets of diagrams produced can be classified as to which of the five possi- ble relationships between the two sets, $\mathrm{A}$ and $\mathrm{C}$, they depict. These relationships can be seen in Figure 1, where they are represented by Euler circles. To illustrate, a set of six diagrams where there was no co-occurrence of property $\mathrm{A}$ and property $\mathrm{C}$ would correspond to a disjoint relationship; a set of six diagrams in which $A$ and $C$ always co-occurred would be an identity relationship.

The number of such relationships constructed for each syllogism in the experimental condition is shown in Table 4. Participants could, in their three sets of diagrams, have presented three different relationships for each syllogism. It can be seen from this table that for each syllogism, there is in most cases a clear preference for one of the relationships over the others, even when several different possibilities exist. The number of different relationships constructed for the different types of syllogisms did not vary as much as might have been expected; singlemodel syllogisms produced 1.4 on average, multiplemodel syllogisms produced 1.9 , and indeterminate produced 2.0. This difference was statistically significant $[F(2,60)=20.83, p<.001]$, but this is primarily attrib-

Table 4

Number of Different Euler Circle Representations Constructed in Experiment 2

\begin{tabular}{lccccccc}
\hline \multicolumn{1}{c}{ Type } & Syllogism & Identity & A Sub C & C Sub A & Overlap & Disjoint & Other \\
\hline \multirow{2}{*}{ Single } & IabAbc & 0 & $12^{*}$ & $13^{*}$ & $66^{*}$ & $1^{*}$ & 1 \\
& AabEbc & 0 & 0 & 3 & 6 & $65^{*}$ & 19 \\
\multirow{5}{*}{ Multiple } & IabEbc & 0 & 0 & $29^{*}$ & $28^{*}$ & $21^{*}$ & 15 \\
& Eablbc & 2 & $5^{*}$ & 7 & $50^{*}$ & $23^{*}$ & 6 \\
& AabIbc & $2^{*}$ & $9^{*}$ & $21^{*}$ & $43^{*}$ & $14^{*}$ & 4 \\
& EabIbc & $0^{*}$ & $4^{*}$ & $18^{*}$ & $43^{*}$ & $22^{*}$ & 6 \\
\hline
\end{tabular}

*This diagram is logically valid for this syllogism. ${ }^{+}$Other responses were those that did not contain one of the end terms (i.e., a or c). 
Table 5 Accuracy Data for Multiple-Model Syllogisms Used in Experiment 3

\begin{tabular}{lccc}
\hline & & & \multicolumn{2}{c}{ Proportion Correct } \\
\cline { 3 - 4 } Syllogism & Correct Answer & Control & Experimental \\
\hline IabEbc & Oac & .25 & .31 \\
EabAbc & Oca & .00 & .06 \\
Eablbc & Oca & .00 & .13 \\
AbaEcb & Oac & .06 & .19 \\
IbaEcb & Oac & .13 & .13 \\
EbaIcb & Oca & .06 & .19 \\
IabEcb & Oac & .31 & .06 \\
EabIcb & Oca & .00 & .19 \\
AbaEbc & Oac & .00 & .25 \\
IbaEbc & Oac & .31 & .25 \\
EbaAbc & Oca & .00 & .25 \\
Ebalbc & Oca & .06 & .12 \\
$M$ & & .10 & .18 \\
\hline
\end{tabular}

utable to Syllogism 2 (AabEbc), for which only one relationship was logically permissible. The other syllogisms produced similar numbers of distinct diagrams.

It was mentioned already that the number of Euler relationships in the diagrams is not the same as the number of mental models, though the two are related. Basically, only some of the Euler circle relationships are regarded as conceptually distinct from each other. The crucial factor is whether a relationship can falsify a putative conclusion. For example, if the provisional conclusion, based on an identity relationship, is that "All A are C," then the relationship in which $\mathrm{A}$ is a subset of $\mathrm{C}$ is not a conceptually distinct model since it cannot falsify the conclusion. The number of mental models for each of our syllogisms can be gleaned from Johnson-Laird and Byrne (1991) for valid syllogisms and from JohnsonLaird and Bara (1984) for indeterminate syllogisms.

According to mental models theory, one would expect that those participants who constructed several mental models would be more accurate in solving syllogisms than those who constructed few. This was tested by calculating the correlation between the number of conceptually distinct models constructed and accuracy on multiple-model and indeterminate syllogisms (it could be claimed that no such correlation would be expected with single-model syllogisms). The Pearson correlation between number of Euler relationships and accuracy was -.31 and that between number of mental models and accuracy was -.14 . Neither of these correlations was statistically significant $(p>.05)$, and in fact both were in the nonpredicted direction.

It is also of interest to examine how accurate the diagrams were as representations of the syllogisms. One of the most common "errors" was to produce diagrams that omitted one of the three terms in the syllogism. Those that omitted one of the end terms are indicated in the final column of Table 4 . These are, however, only errors of omission, since the majority of these representations were not inconsistent with the premises. Diagrams that actually contradicted the premises were rare.

\section{Discussion}

The present findings hold little comfort for proponents of mental models theory. There is no evidence that encouraging people to construct alternative models, as happened in the diagram-drawing condition, led to any improvement in accuracy. Furthermore, although participants seemed perfectly capable of producing conceptually distinct models, there was little difference in the number constructed for single- and multiple-model syllogisms. Indeed, one of the two single-model syllogisms produced as many models as the two multiple-model ones. Furthermore, there was no evidence that the number of conceptually distinct models drawn correlated with accuracy on the syllogism task.

The present experiment can be criticized for the small number of syllogisms used. In particular, there were only two multiple-model syllogisms in this study. These are syllogisms in which attempted falsification is claimed to take place, and hence it is important to know whether similar results would be obtained with a larger sample of such syllogisms. It is also important to know whether there is a correlation between number of conceptually distinct diagrams drawn and reasoning accuracy when a larger sample is used. Experiment 3 was essentially a replication of Experiment 2 using only multiple-model syllogisms. The predictions were that drawing models of the premises would facilitate performance on these syllogisms and that the number of different models drawn would correlate with overall accuracy.

\section{EXPERIMENT 3}

\section{Method}

Participants. The 32 participants were 1st-year undergraduate psychology students from the University of Plymouth who received course credit for their participation.

Materials. The 16 syllogisms used were all valid. Twelve of them were multiple model, and 4 were single model; the latter were selected as filler items to avoid response sets, given that the correct response to the 12 multiple-model syllogisms is always "some ... not." The syllogisms were selected from all four figures but no attempt was made to have equal numbers of each figure. The forms of syllogism used can be seen in Table 5 .

Similar material to that of Experiment 2 was used-that is, shapes (squares or circles), patterning (striped or cross-hatched), and borders (bold or faint). Two different versions of the material were used to defend against the occurrence of any material-specific effects. Half of the participants received syllogisms in which the A term was shape, the $B$ term was border, and the $C$ term was pattern, while the other half received syllogisms in which the content of the $\mathrm{B}$ and $\mathrm{C}$ terms was reversed.

Procedure. The procedure was similar to that of Experiment 2. The 16 participants in the control condition were presented with a paper booklet containing the 16 syllogisms in random order. Their task was simply to solve the syllogisms. The 16 participants in the experimental group were required to draw three sets of six shapes consistent with each syllogism. They were presented with the syl- 
Table 6

Number of Different Euler Circle Representations Constructed in Experiment 3

\begin{tabular}{lllllllllllll}
\hline Syllogism & \multicolumn{1}{l}{ Identity } & \multicolumn{3}{c}{ A Sub C } & \multicolumn{2}{c}{ C Sub A } & \multicolumn{2}{c}{ Overlap } & \multicolumn{2}{c}{ Disjoint } & \multicolumn{2}{c}{ Other $^{\dagger}$} \\
\hline IabEbc & 0 & $(1)$ & 2 & $(0)$ & $7^{*}$ & $(11)$ & $14^{*}$ & $(19)$ & $7^{*}$ & $(7)$ & 18 & $(6)$ \\
EabAbc & 0 & $(0)$ & $1^{*}$ & $(3)$ & 2 & $(0)$ & $32^{*}$ & $(25)$ & $13^{*}$ & $(14)$ & 0 & $(2)$ \\
EabIbc & 0 & $(0)$ & $5^{*}$ & $(2)$ & 5 & $(5)$ & $25^{*}$ & $(25)$ & $11^{*}$ & $(10)$ & 2 & $(1)$ \\
AbaEcb & 0 & $(0)$ & 0 & $(0)$ & $13^{*}$ & $(4)$ & $18^{*}$ & $(7)$ & $12^{*}$ & $(14)$ & 5 & $(12)$ \\
IbaEcb & 0 & $(0)$ & 1 & $(0)$ & $11^{*}$ & $(11)$ & $23^{*}$ & $(21)$ & $7^{*}$ & $(8)$ & 6 & $(12)$ \\
EbaIcb & 0 & $(0)$ & $11^{*}$ & $(2)$ & 2 & $(0)$ & $19^{*}$ & $(18)$ & $9^{*}$ & $(10)$ & 7 & $(2)$ \\
IabEcb & 0 & $(1)$ & 0 & $(0)$ & $16^{*}$ & $(6)$ & $17^{*}$ & $(21)$ & $11^{*}$ & $(11)$ & 5 & $(3)$ \\
EabIcb & 0 & $(0)$ & $4^{*}$ & $(3)$ & 1 & $(2)$ & $30^{*}$ & $(26)$ & $10^{*}$ & $(12)$ & 3 & $(4)$ \\
AbaEbc & 0 & $(0)$ & 1 & $(0)$ & $9^{*}$ & $(7)$ & $15^{*}$ & $(11)$ & $14^{*}$ & $(17)$ & 9 & $(6)$ \\
IbaEbc & 0 & $(0)$ & 0 & $(0)$ & $5^{*}$ & $(10)$ & $19^{*}$ & $(13)$ & $7^{*}$ & $(14)$ & 17 & $(9)$ \\
EbaAbc & 0 & $(0)$ & $7^{*}$ & $(2)$ & 0 & $(2)$ & $23^{*}$ & $(19)$ & $10^{*}$ & $(10)$ & 8 & $(5)$ \\
EbaIbc & 0 & $(0)$ & $1^{*}$ & $(3)$ & 1 & $(3)$ & $23^{*}$ & $(25)$ & $20^{*}$ & $(6)$ & 3 & $(3)$ \\
\hline
\end{tabular}

Note-Figures in parentheses are from Experiment 4. *This diagram is logically valid for this syllogism. ${ }^{+}$Other responses were those that did not contain one of the end terms (i.e., a or c) or failures to complete all diagrams.

logism at the top of the page, below which were three empty grids of six squares. In each square of each grid, they were asked to draw a shape in such a way that each set of six shapes was consistent with the two premises of the syllogism. Participants were asked to make each set different. On completion of the three sets of shapes, participants were required to solve the syllogism in the same way as the control group.

\section{Results}

The accuracy data for the control and experimental conditions can be seen in Table 5. It is clear that the accuracy levels were low for all the syllogisms. Accuracy data were analyzed with an ANOVA in which the factors were syllogism (12) and condition (control or experimental). There was a main effect of syllogism $[F(11,330)=$ $2.79, p<.01]$, but no main effect of condition $[F(1,30)=$ $.86, p>.1]$; the interaction between syllogism and condition was not significant $[F(11,330)=1.65, p<.05]$.

In just the same way as in Experiment 2, it was possible to analyze the number of different Euler circle relationships present in the diagrams drawn in the experimental condition, and also the number of different mental models that map onto these. The figures for Euler circle relationships are presented in Table 6 . The correlation between accuracy on the 12 syllogisms and number of different Euler relationships was - $.35(p>.1)$, while that between accuracy and number of mental models was -.41 $(p>.1)$. It might be objected that this is an unfair test of mental models theory since there might be a small number of participants who both produced many models and gave the wrong answer, thus reducing the correlation that might exist in the other participants. However, even if these correlations are done at the level of individual participants, none of the correlations turn out to be significant, and considerably more than half are negative.

As can be seen from Table 6, most of the diagrams drawn were consistent with the information presented in the premises. Fewer than $4 \%$ of the classifiable diagrams were in conflict with the premises. In addition, the conclusions that participants made, whether logically correct or not, were largely consistent with the diagrams drawn. In $98 \%$ of the cases, the propositional conclusion given was consistent with at least one of the diagrams drawn, and in $80 \%$ of the cases, the conclusion was compatible with all the classifiable drawings.

\section{Discussion}

The results of this experiment are clear-cut. Not one of the predictions made by mental models theory with respect to falsification was confirmed. There was no evidence that requiring participants to produce diagrams of their mental representations led to any improvement in performance, even though the instructions stressed the need to produce as many different representations as possible. Further, there was no evidence that the richness of these representations, whether measured in terms of the number of mental models or the number of Euler relationships, correlated with overall accuracy. There is thus no evidence to support the contention that the construction of falsifying models is a central part of syllogistic reasoning.

A possible objection to Experiments 2 and 3 is that the diagram task contained certain demand characteristics. Participants were required to fill in precisely three matrices, each containing six boxes. This task might encourage participants to construct as many models as possible, even with single-model syllogisms, which might explain why there was no difference between single- and multiplemodel syllogisms in Experiment 2. Another quite different possibility is that participants solving multiplemodel syllogisms might have been restricted in the number of conceptually distinct models they constructed by being limited to drawing just three sets of diagrams. The diagram task can also-and perhaps more importantlybe criticized on the grounds that it might have been treated by participants as completely separate from the syllogism task; participants might have carried out the diagram task and then solved the syllogism without utilizing the information contained in the diagrams. It was for such reasons that Experiment 4 was conducted. This was es- 
sentially a repeat of the experimental condition of Experiment 3, but participants were allowed to construct as many diagrams as they wished and the instructions stressed that the diagrams should be used in solving the syllogisms.

\section{EXPERIMENT 4}

\begin{abstract}
Method
Participants. There were 22 participants in this study who took part either for financial reward or for course credit.

Materials. Exactly the same syllogisms were used as in Experiment 3 . The task differed in two ways. First, participants were asked to draw one set of six shapes for each syllogism and then to complete as many other sets as they wished. In total, 13 empty matrices were provided, on the (correct) assumption that no participants would require this many. Second, the instructions stressed throughout that the diagrams were to be used in solving the syllogism. The instructions stated early on: "You should try to draw pictures that you think will help you in solving the syllogism," and this point was repeatedly emphasized.
\end{abstract}

Procedure. Participants were run in exactly the same way as in the experimental condition of Experiment 3.

\section{Results}

The main aim of the present experiment was to investigate whether the two manipulations-allowing participants to fill in as many matrices as they liked and stressing the use of the diagram task in solving the syllogismswould alter the pattern of results obtained in Experiment 3 . The results of the diagram task are presented in parentheses in Table 6 . It is clear that the pattern of diagrams produced was similar to that in the experimental group of Experiment 3; although there are one or two minor exceptions, in general the same relationships were preferred with each syllogism. It is also worth noting that the average number of diagrams produced was actually lower than in Experiment 3, with participants in the present study producing on average 1.9 ; in Experiment 3 , participants were required to produce three diagrams.

The correlation between accuracy and the number of conceptually different diagrams produced was low. With number of Euler relationships, the correlation was .22, while with number of mental models it was .14. Neither of these correlations approached significance.

Experiment 4 was not designed to investigate differences between single- and multiple-model syllogisms, but post hoc comparisons are possible given that all four of the filler syllogisms were single-model ones. Accuracy was, of course, much higher on these (.41 vs. .03), but the finding of most interest is that the number of conceptually different relationships produced was, at 1.9 , identical to that for multiple-model syllogisms.

\section{Discussion}

Experiment 4 confirmed the results of Experiment 3. Even when participants were allowed to construct as many diagrams as they wished, there was no suggestion of any correlation between the number of diagrams constructed and accuracy in solving multiple-model syllogisms. Par- ticipants produced fewer diagrams in this experiment than in Experiment 3, suggesting that Experiment 3 did not unduly constrain participants in the number of relationships they could construct; on the contrary, Experiment 3 would appear to have forced participants to produce more diagrams than they would have done spontaneously.

The instructions in Experiment 4 stressed the importance of the diagram task in helping to solve the syllogisms, but this appears to have had no effect. A post hoc analysis showed that the present manipulations did not produce any difference between single- and multiplemodel syllogisms in terms of the number of conceptually different diagrams produced. The results thus indicate that the findings of Experiments 3 and 4 are robust.

\section{GENERAL DISCUSSION}

The principal aim of the present series of experiments was to investigate whether reasoners try to construct alternative models of syllogisms in an attempt to falsify conclusions they have tentatively derived. All of the findings suggest that this is not the case. In Experiment 1, there was no indication that reasoners derived any more possible conclusions for multiple-model syllogisms than they did for single-model ones. In the first three experiments, there was no indication that participants who were required to indicate provisional conclusions or to draw representations of the premises produced any more correct responses than did control participants. Nor was there in any of the experiments a correlation between accuracy and the number of conclusions considered or the number of different diagrams constructed.

Despite this generally negative evidence, all the evidence suggests that multiple-model syllogisms are more difficult than single-model ones. This was found in both Experiments 1 and 2, and these in turn simply replicate a well-established finding (see, e.g., Johnson-Laird \& Byrne, 1991). Valid, multiple-model syllogisms (the majority of which were used in Experiments 3 and 4) are extraordinarily difficult. What is more, although mental models theory is not the only one that predicts the difficulty of these syllogisms (see Evans et al., 1993), it is one of the more attractive theories in terms of its psychological plausibility. We will return to the discussion of mental models theory after briefly considering the implications of the present findings for other theories of syllogistic reasoning.

A number of alternative approaches claim to be related to mental models. Stenning and his colleagues (e.g., Stenning \& Yule, 1997) have proposed that syllogistic reasoning can be characterized as identifying critical individuals who must exist given the premises. They pointed out that, at an abstract level, this is equivalent to constructing mental models (and, indeed, equivalent to other methods of solving syllogisms). It is not easy to make specific predictions about the tasks used in the present studies on the basis of this proposal. However, it might have been expected that requiring reasoners to draw di- 
agrams of the premises would improve performance, since this should facilitate identification of a critical individual. However, the results of Experiments 2 and 3 indicate that this was not the case.

Polk and Newell (1995), too, have pointed out the similarities between their theory and that of mental models. The main difference is, as discussed earlier, that Polk and Newell construe syllogistic reasoning as involving only verbal processes, and see no reason to postulate a process of falsification. In this respect, the present findings are clearly supportive of their ideas. Polk and Newell have claimed that mental models without falsification reduces to their verbal reasoning theory. A possible problem for their theory, however, is that reasoners do consider alternative conclusions. The theory assumes that people generate propositions until a legal conclusion is produced. When this occurs, processing stops and the conclusion is given as the answer. The results of Experiment 1 indicate that participants considered, on average, at least one alternative conclusion, which on Polk and Newell's analysis should not have occurred. Nevertheless, the present results will hold considerable comfort for proponents of this theory.

The present finding that falsification does not take place provides some comfort to those who argue that people engage in little logical reasoning, but instead base their responses on surface characteristics of the premises. The foremost of these theories is currently Chater and Oaksford's (in press) rational choice theory, which suggests that syllogistic reasoning responses are determined by the informativeness of the premises. The conclusions our participants produced are largely consistent with their predictions. In Experiment 1, for example, the direction of the conclusion on the 11 syllogisms with an "all" premisethat is, those on which people should be able to draw conclusions with confidence-corresponded to their predictions in 10 cases, with no difference in the other case. We also found, as they would have predicted, no difference in accuracy between the different syllogistic figures. This was already reported for Experiment 1, where figure was an independent variable, and it was also found in Experiment 3, where a post hoc analysis revealed that mean accuracy was virtually identical across figures $(F<$ 1). It is worthy of note that some of Chater and Oaksford's figural predictions are risky in the sense that they go against the effects predicted by mental models theory. In this respect, then, the present findings favor rational choice theory over mental models theory.

In other ways, however, our findings sit uncomfortably with Chater and Oaksford's (in press) predictions. Their theory predicts that participants will be confident in their conclusion when one of the premises contains "all," the maximally informative quantifier. One might, then, have predicted that in Experiment 1 participants would have considered fewer conclusions when one of the premises was "all" than in other cases. Although there was a small difference in the predicted direction, with "all" premises leading to 1.05 other conclusions considered compared with 1.13 for other quantifiers, this difference did not ap- proach statistical significance $[t(19)=1.26, p>.1]$. In addition, there was, in Experiment 3, a close correspondence between the diagrams drawn and the conclusions that participants gave. This suggests that there is a link between the way in which the premises are represented and the conclusion reached. Such a link is directly contrary to the tenets of rational choice theory, which claims that heuristics rather than representational processes are at the heart of syllogistic reasoning.

Other current theories of syllogistic reasoning are less easy to test against the present data. Rips's (1994) theory of inference rules does not make any specific predictions concerning the provisional conclusions that might be drawn. It might be argued, however, that the close correlation between diagrams and conclusions in Experiment 3 is difficult to explain using this theory, which does not clearly specify the mental representation involved in syllogistic reasoning. Ford's (1994) theory claims that there are individual differences in syllogistic reasoning. Our findings certainly attest to the variety of different conclusions that participants reach, but beyond that seem to bear little on specific predictions derivable from her theory.

It seems fair to conclude that our results, while posing problems for mental models theory, also call into question other theories of syllogistic reasoning. It is important to remember that only one aspect of mental models theoryfalsification-has been challenged, and to consider what happens to mental models theory if the notion of falsification is removed. It may, in fact, lose little of its explanatory power if it is assumed that reasoners construct just a single model rather than constructing a preliminary model that they then try to falsify (Polk, 1993). The single model that is constructed for multiple-model syllogisms will often lead to conclusions that are not valid, since alternative models need to be constructed to preclude many of the conclusions that might be generated. This is precisely what reasoners do when confronted with multiplemodel syllogisms: produce conclusions compatible with one (but not all) possible models.

The assumption that reasoners only construct a single model seems to encounter an insuperable difficulty, since all single models lead to one (or more) valid conclusions. Hence how can people ever decide that no valid conclusion follows? In fact, people say that no valid conclusion follows rather less often than they should, a phenomenon that has been called the preference for propositional conclusions (Revlis, 1975). Nevertheless, it is a response that is regularly given and hence must be explained. Perhaps it occurs whenever participants fail to find a conclusion easily, being almost akin to a "don't know" response. Or perhaps it is given when participants know that other ways of representing the premises are possible, which could well eliminate the conclusion(s) they are considering, but lack the mental capacity to construct such representations. In either case, a "no valid conclusion" can be given even when only one model has been constructed. A not dissimilar notion has been proposed by Polk and Newell (1995), who suggested that "no valid 
conclusion" responses are given when verbal reasoning processes have been exhausted.

It is also appropriate in the context of the present experiments to consider just what is meant by "conceptually distinct mental models." All syllogisms can be classified as to the number of mental models they permit, but the rationale for this is not always transparent. Consider, for example, Syllogism 1 in Experiment 2: "Some A are B; all B are C." As can be seen in Table 4, this permits four different Euler circle relationships, but was regarded by Johnson-Laird and Byrne (1991) as a single-model syllogism. In one situation, it clearly is a single-model syllogism. If a reasoner builds a model and draws the tentative conclusion that "Some A are C," then there is no alternative model that can be constructed that will falsify this conclusion. On the other hand, however, if the provisional conclusion had been reached that "All A are C"-a conclusion that is valid with some representations of the premises - then it is possible to construct models that will falsify this conclusion. Does this mean that this syllogism is single model in some situations and multiple model in others, depending on which representation is constructed first? This may well be the case, but it is not a possibility that has been considered in previous discussions of mental models theory.

Under the proposal being put forward here, this paradox is resolved. Reasoners are more likely to produce certain representations than others (see, e.g., Tables 4 and 6). If the initial model constructed is one that leads to a valid conclusion, then this will be a simple syllogism, irrespective of how many other models are conceptually possible. On the other hand, if the preferred model is one that leads to invalid conclusions, then the syllogism will produce many errors. This proposal may not be too far away from Johnson-Laird and Byrne's (1993) suggestion that reasoners may be rational in principle but not necessarily when carrying out reasoning problems. At the heart of our proposal is the suggestion that reasoners are able to construct alternative models-for example, when their first model leads to an unbelievable conclusionbut that they normally construct only one.

If this suggestion is correct, then future research should focus not on comparing single- and multiple-model syllogisms, but on ascertaining what is the preferred representation resulting from certain types of premise pairs, and why. The key issue would appear to be how the information in the two premises is combined into a single representation, not how different representations are constructed in an attempt to falsify preliminary conclusions. Mental models theory can provide an account of how premises are combined, but so far has not indicated specifically how this is done, and hence which is the first (and in our account, the only) model constructed. A more complete model of this process is required before the adequacy of the theory in explaining syllogistic reasoning can be fully assessed.

\section{REFERENCES}

Byrne, R. M. J., \& Johnson-LaiRd, P. N. (1990). Remembering conclusions we have inferred. In J. P. Caverni, J.-M. Fabre, \& M. Gonzalez (Eds.), Cognitive biases (pp. 109-120). Amsterdam: Elsevier, North-Holland.

ChATER, N., \& OAKsford, M. (in press). Rational analysis and heuristic processes for syllogistic reasoning. Cognitive Psychology.

Evans, J. St. B. T., Newstead, S. E., \& BYRNe, R. M. J. (1993). Human reasoning: The psychology of deduction. Hillsdale, $\mathrm{NJ}$ : Erlbaum.

FoRD, M. (1994). Two modes of representation and problem solution in syllogistic reasoning. Cognition, 4, 1-71.

JANIS, I., \& FRICK, F. (1943). The relationship between attitudes towards conclusions and errors in judging logical validity of syllogisms. Journal of Experimental Psychology, 33, 73-77.

JoHNSON-LAIRD, P. N., \& BARA, B. G. (1984). Syllogistic inference. Cognition, 16, 1-61.

JohnSON-LAIRd, P. N., \& BYRNe, R. M. J. (1991). Deduction. Hillsdale, $\mathrm{NJ}$ : Erlbaum.

Johnson-Laird, P. N., \& Byrne, R. M. J. (1993). Models and deductive rationality. In K. I. Manktelow \& D. E. Over (Eds.), Rationality: Psychological and philosophical perspectives (pp. 177-210). London: Routledge \& Kegan Paul.

Newstead, S. E., Pollard, P., Evans, J. St. B. T., \& Allen, J. (1992). The source of belief bias in syllogistic reasoning. Cognition, $45,257-$ 284.

OAKHill, J., Johnson-Laird, P. N., \& Garnham, A. (1989). Believability and syllogistic reasoning. Cognition, 31, 117-140.

OAKSFORD, M., \& CHATER, N. (1994). A rational analysis of the selection task as optimal data selection. Psychological Review, 101, 608-631.

PolK, T. A. (1993). Mental models, more or less. Behavioral \& Brain Sciences, 16, 362-363.

Polk, T. A., \& Newell, A. (1995). Deduction as verbal reasoning. Psychological Review, 102, 533-566.

REVLIS, R. (1975). Syllogistic reasoning: Logical decisions from a complex data base. In R. J. Falmagne (Ed.), Reasoning: Representation and process in children and adults (pp. 93-133). Hillsdale, NJ: Erlbaum. RIPS, L. J. (1994). The psychology of proof. Cambridge, MA: MIT Press.

STENNING, K., \& YUle, P. (1997). Image and language in human reasoning: A syllogistic illustration. Cognitive Psychology, 34, 109-159. WOODWORTH, R. S., \& SELLS, S. B. (1935). An atmosphere effect in syllogistic reasoning. Journal of Experimental Psychology, 18, 451-460.

(Manuscript received February 7, 1997; revision accepted for publication February 19, 1998.) 